\title{
Neuropeptides centraux et régulations hydrique et cardiovasculaire
}

UMR 7241 / U 1050 (CIRB)

\section{Catherine Llorens-Cortes}

\section{(2) OpenEdition}

\section{Journals}

\section{Édition électronique}

URL : https://journals.openedition.org/annuaire-cdf/1084

DOI : 10.4000/annuaire-cdf. 1084

ISBN : 978-2-7226-0325-7

ISSN : 2109-9227

\section{Éditeur}

Collège de France

\section{Édition imprimée}

Date de publication : 1 avril 2013

Pagination : 880-884

ISBN : 978-2-7226-0198-7

ISSN : 0069-5580

\section{Référence électronique}

Catherine Llorens-Cortes, « Neuropeptides centraux et régulations hydrique et cardiovasculaire », L'annuaire du Collège de France [En ligne], 112 | 2013, mis en ligne le 22 novembre 2013, consulté le 22 août 2022. URL : http://journals.openedition.org/annuaire-cdf/1084 ; DOI : https://doi.org/10.4000/ annuaire-cdf.1084 


\title{
Neuropeptides centraux et régulations hydrique et cardiovasculaire UMR 7241 / U 1050 (CIRB)
}

\author{
Responsable : Catherine LLORENS-CORTES \\ Équipe : Xavier ITURRIOZ, Bernard MaIGRET, Yannick MARC, Laurence BODINEAU, \\ Alain Frugière, Romain Gerbier, Annette Hus-Citharel, Bo LI, Gao Jl, \\ Émilie Ceraudo, Cécile Galanth, Hani Aboukhamis, Clara Pouchelon, \\ Rodrigo Alvear-Perez, Nadia PIcco-de Mota
}

\section{RECHERCHE}

\section{Le système rénine-angiotensine (SRA) cérébral}

Nous avons montré dans ce système que l'aminopeptidase A (APA) est impliquée dans la conversion de l'angiotensine (Ang) II en AngIII ; développé les premiers inhibiteurs spécifiques et sélectifs de l'APA, inexistants jusqu'à ce jour, et identifié le peptide effecteur du SRA cérébral qui est l'AngIII et non l'AngII comme établi à la périphérie. L'AngIII au niveau central exerce un effet stimulateur tonique sur le contrôle de la pression artérielle (PA) chez le rat hypertendu. Ainsi le blocage central et non systémique de l'APA diminue fortement la PA dans différents modèles expérimentaux d'hypertension artérielle (HTA), suggérant que l'APA cérébrale constituerait une cible thérapeutique potentielle pour le traitement de certaines formes d'HTA. L'HTA touche près de $20 \%$ de la population et représente un facteur de risque majeur de nombreuses maladies telles que les affections coronariennes, les accidents vasculaires cérébraux, l'insuffisance cardiaque et l'insuffisance rénale. L'importance de cette maladie a justifié le développement de nombreuses familles thérapeutiques, cependant, elle reste difficile à contrôler. Il y a donc un besoin de thérapies complémentaires. Notre projet vise à développer un nouvel antihypertenseur avec un mode d'action différent de ceux utilisés jusqu'à présent. Pour cela, nous avons développé, en collaboration avec l'équipe du Pr B.P. Roques (INSERM U 1022), de nouveaux inhibiteurs de l'APA, puissants et sélectifs capables de passer les barrières intestinale, hépatique et hématoencéphalique après administration par voie orale, exemplifié par le RB150 (1 brevet INSERM/CNRS avec une licence exclusive avec la société Quantum Genomics) qui, après administration par voie orale chez le rat hypertendu DOCA-sel (hypertension dépendante du sel) ou le rat spontanément hypertendu SHR (un modèle de l'hypertension essentielle humaine), pénètre dans le cerveau, inhibe l'activité du SRA cérébral et a un effet hypotenseur qui dure plusieurs heures. Cet effet est dû en partie à une baisse de la sécrétion de vasopressine (AVP), qui provoque une augmentation de la diurèse aqueuse et diminue ainsi le volume sanguin contribuant à ramener la PA à une valeur physiologique et, d'autre part, à une baisse du tonus sympathique qui aboutit à une diminution des résistances vasculaires et à une baisse de la PA. Nous poursuivons ce programme de recherche en partenariat avec la société Quantum Genomics. Les études précliniques réglementaires de toxicologie, de pharmacocinétique et de pharmacologie de sécurité ont permis de démontrer l'innocuité du RB150/QGC001 chez le rat et le chien à des 
doses supérieures à 50 fois la dose présentant une activité anti-hypertensive chez le rat, ainsi qu'une bonne biodisponibilité du produit chez le chien (communiqué de presse de Quantum Genomics du $1^{\mathrm{er}}$ avril 2011). Le RB150 a reçu en janvier 2012 l'avis favorable du Comité de protection des personnes d'île-de-France (CPP IdF VIII) puis celui de l'AFSSAPS en février 2012 pour le premier essai clinique chez l'Homme. Un communiqué de presse, en avril 2012, a annoncé le lancement, dans le cadre d'une collaboration avec notre équipe, ainsi qu' avec celle du Pr B.P. Roques et le Centre d'investigation clinique (CIC) de l'hôpital européen Georges Pompidou (HEGP), dirigé par le Pr. M. Azizi, du premier essai clinique phase 1 chez le volontaire sain avec le RB150/QGC001 (http://www.quantum-genomics.com/news_ read.php?txtid=33). Les résultats de cette étude seront disponibles en 2013. Le RB150 pourrait ainsi constituer le prototype d'une nouvelle classe d'agents antihypertenseurs à action centrale, actif par voie orale, qui ouvre une nouvelle voie de régulation de la pression artérielle. À l'heure actuelle les traitements contre l'hypertension agissent sur les vaisseaux, le cœur ou l'élimination d'eau et de sel dans l'urine et, deux voire trois traitements sont utilisés pour normaliser la pression artérielle. L'avantage du traitement par le RB150 est qu'avec un seul traitement, en bloquant le SRA cérébral, on agira sur ces trois organes en contrôlant la sécrétion de vasopressine, l'activité sympathique et l'activité du baroréflexe. L'ensemble de cette recherche qui a démarré en 1993 pourrait aboutir au développement d'un nouveau médicament antihypertenseur à action centrale. Une ANR BiotecS (20092012) soutient ce projet.

\section{Le système apélinergique}

En recherchant un récepteur spécifique de l'Ang III, nous avons isolé chez le rat un récepteur couplé aux protéines $\mathrm{G}$, partageant $95 \%$ d'identité de séquence avec le récepteur orphelin humain APJ qui s'est révélé être le récepteur d'un nouveau peptide, l'apéline. Nous avons caractérisé pharmacologiquement ce récepteur, établi sa distribution, ainsi que celle des neurones apélinergiques dans le cerveau de rat. Nous avons observé que les neurones ocytocinergiques magnocellulaires et parvocellulaires de l'hypothalamus expriment l'ARNm du récepteur de l'apéline de même que l'apéline (pour 20\% d'entre eux). Par une double approche, immunohistochimique d'une part (détection de c-Fos) et électrophysiologique d'autre part, nous avons montré que l'apéline administrée par voie intracérébroventriculaire inhibe l'activité des neurones ocytocinergiques, une donnée corrélée avec une diminution de la quantité de lait délivrée aux nouveau-nés. Ces données suggèrent un rôle de l'apéline dans la régulation de l'activité des neurones ocytocinergiques de l'hypothalamus au cours de l'allaitement.

Par ailleurs, l'apéline et son récepteur sont co-exprimés avec l'AVP dans les neurones magnocellulaires vasopressinergiques. Nous avons mis en évidence que l'apéline, injectée par voie centrale chez la rate en lactation, diminue l'activité électrique phasique de ces neurones et la sécrétion d'AVP dans la circulation sanguine, provoquant une diurèse aqueuse. Enfin nous avons établi chez le rat déshydraté que l'apéline et l'AVP sont régulées de façon opposée afin de maintenir l'équilibre hydrique de l'organisme, en optimisant la sécrétion d'AVP dans la circulation sanguine et évitant ainsi une perte d'eau supplémentaire par les reins. Afin de poursuivre cette exploration chez l'homme, nous avons réalisé, en 
collaboration avec le CIC de l'HEGP, la première étude clinique sur l'apéline chez le volontaire sain. Nous avons montré que les sécrétions d'apéline et d'AVP sont régulées de façon opposée par les stimuli osmotiques et volémiques, suggérant que l'apéline comme l'AVP joue un rôle crucial dans le maintien de l'équilibre hydrique chez l'Homme comme chez le rongeur. Afin de compléter les données sur le mode d'action de l'apéline dans le maintien de l'équilibre hydrique, plusieurs études ont été effectuées dans le laboratoire et suggèrent que l'effet aquarétique de l'apéline ne serait pas uniquement dû à un effet central via une inhibition de la sécrétion d'AVP dans le sang mais pourrait aussi impliquer un effet direct au niveau du rein via une augmentation du flux sanguin rénal et/ou en antagonisant l'effet de l'AVP au niveau des canaux collecteurs où les récepteurs de l'apéline et de l'AVP sont co-exprimés. En effet, dans la partie médullaire du canal collecteur, l'apéline inhibe la production d'AMPc induite par la dDAVP, un agoniste des récepteurs $V_{2}$ de l'AVP. Cette inhibition de la production d'AMPc est directement reliée à une inhibition de l'insertion des aquaporines de type 2 à la membrane apicale induisant ainsi un effet diurétique. L'apéline joue également un rôle important dans le contrôle des fonctions cardiovasculaires : injectée par voie intraveineuse elle diminue la PA et augmente la force contractile du myocarde par un effet inotrope positif.

Le récepteur de l'apéline apparaît donc être une cible thérapeutique potentielle pour le traitement des maladies cardiovasculaires et des rétentions hydriques. Grâce à une collaboration avec le laboratoire du Pr. M. Hibert (UMR CNRS 7200) nous avons isolé le premier agoniste non peptidique du récepteur de l'apéline l'E339-3D6 qui présente une affinité de $100 \mathrm{nM}$ pour le récepteur de l'apéline. Ce composé est un agoniste partiel sur l'activation du couplage à la protéine Gai et un agoniste complet sur l'internalisation du récepteur. Ex vivo, ce composé est capable d'induire, comme l'apéline, une vasorelaxation des anneaux aortiques de rat pré-contractés par la noradrénaline comparable à celle induite par l'acétylcholine. In vivo chez la souris, l'E339-3D6 injecté par voie centrale en doses croissantes inhibe de façon dose-dépendante la sécrétion d'AVP, dans la circulation sanguine, induite par la déshydratation avec une efficacité identique à celle de l'apéline. Afin d'optimiser cette molécule, nous avons entrepris des études de relation structure-activité de l'E339-3D6. Ceci nous a permis de définir les éléments structuraux nécessaires à son affinité, sa capacité à activer les voies de signalisation ou à induire l'internalisation du récepteur de l'apéline. Par ailleurs, nous avons entrepris une campagne d'études structure-fonction du récepteur de l'apéline par modélisation moléculaire et mutagénèse dirigée dans le but de définir les éléments structuraux nécessaires à la liaison de l'apéline à son récepteur et à son activation. Pour cela nous avons construit, en collaboration avec le Dr B. Maigret (LORIA, Nancy), un modèle tridimensionnel (3D) du récepteur de l'apéline complexé avec différents fragments d'apéline. Nous avons visualisé plusieurs interactions entre le ligand et son récepteur. L'une d'entre elles correspond à une poche aromatique qui accueille la phénylalanine C-terminale de l'apéline. La caractérisation par mutagénèse dirigée des résidus (Phe 255 et Trp 259) constituant cette poche a montré que ces résidus situés dans l'hélice VI du récepteur de l'apéline, bien que ne jouant pas de rôle dans l'affinité de l'apéline pour son récepteur ou encore dans l'activation du couplage du récepteur à la protéine Gai, sont indispensables à son internalisation. Cette étude représente une première validation du modèle 3D du récepteur de l'apéline qui va pouvoir maintenant être utilisé pour des campagnes de criblage in silico de chimiothèques virtuelles. Ce programme est soutenu par une ANR PCV (2009-2012). 


\section{PUBLICATIONS 2011-2012}

Feng D., Yang S.-K., Loudes C., Simon A., Al-Sarraf T., Culler M., Alvear-Perez R., Llorens-Cortes C., Chen C., Epelbaum J. et Gardette R., «Ghrelin and Obestatin modulate Growth Hormone Releasing Hormone release and synaptic inputs onto GHRH neurons », Eur. J. Neurosci, 34(5), 2011, 732-744.

Bodineau L., Taveau C., Le Quan Sang H.H., Osterstock G., Queguiner I., Moos F., Frugière A. et Llorens-Cortes C., « Data supporting a new physiological role for brain apelin in the regulation of endocrine oxytocin neurons in lactating rats », Endocrinology, 152(9), 2011, 3492-3503.

Duparc T., Colom A., Cani P.D., Massaly N., Rastrelli S., Le Gonidec S., Moulédous L., Frances B., Llorens-Cortes C., Pospisilik J.A., Penninger J.M., Delzenne N.M., Valet P., Castan-Laurell I. et Knauf C., «Central Apelin controls Glucose Homeostasis via a NO dependent pathway: Mechanism Impaired in High-Fat Diet », Antioxid Redox Signal, 15(6), 2011, 1477-1496.

Reaux-Le Goazigo A., Bodineau L., De Mota N., Jeandel L., Chartrel N., Knauf C., Raad C., Valet P. et Llorens-Cortes C., « Apelin and the proopiomelanocortin system: a new regulatory pathway of hypothalamic $\alpha-\mathrm{MSH}$ release », Am. J. Physiol. Endocrinol. Metab., 301(5), 2011, e955-66.

Marc Y. et Llorens-Cortes C., "The role of the brain renin-angiotensin system in hypertension: implications for new treatment », Prog. Neurobiol., 95(2), 2011, 89-103.

Chartrel N., Alonzeau J., Alexandre D., Jeandel L., Alvear R., Vaudry H., Leprince J., Boutin J., Anouar Y. et Llorens-Cortes C., « The RFamide neuropeptide 26RFa and its role in the control of neuroendocrine functions », Front Neuroendocrinol, 32(4), 2011, 389-387.

Galanth C., Hus-Citharel A., Li B. et Llorens-Cortes C., « Apelin in the control of body fluid homeostasis and cardiovascular functions ", Curr. Pharm. Des., 18(6), 2012, 789-798.

Marc Y., Ji G., Balavoine F., Michaud A., Roques B. et Llorens-Cortes C., «Central antihypertensive effects of orally active aminopeptidase a inhibitors in spontaneously hypertensive rats », Hypertension, 18 juin 2012 [doi: 10.1161/HYPERTENSIONAHA.112.190942].

Iturrioz X., O-Wang J., Cooper M.D. et Llorens-Cortes C., « Glutamyl Aminopeptidase », in Barret A., Rawlings N.D. et Woessner J. (éd.), Handbook of proteolytic enzymes, Academic press, 2012, 410-414.

Iturrioz X. et Llorens-Cortes C., « Apelin », in Kastin A.J. (éd.), Handbook for Biologically Active Peptides, Elsevier, 2012, sous presse.

\section{NOUVEAUX BREVETS}

«Combination of (3S,3S') 4,4'-disulfanediylbis(3-aminobutane 1-sulfonic acid) and a second antihypertensive agent ». Brevet français déposé par l'INSERM et la société Quantum Genomics en décembre 2011.

Inventeurs : Llorens-Cortes C. ; la liste des autres inventeurs reste à finaliser.

\section{THĖSES SOUTENUES}

Yannick Marc (2007-2011) : Analyse des propriétés pharmacologiques des inhibiteurs de l'aminopeptidase A, comme agents antihypertenseurs à action centrale. Thèse de doctorat en sciences de l'université René Descartes, Paris V, école doctorale «Médicament, toxicologie, chimie et environnement », mention très honorable. 
Romain Gerbier (2008- 2011): Études structure-fonction du récepteur de l'apéline par modélisation moléculaire et mutagenèse dirigée. Recherche d'agonistes et/ou d'antagonistes de ce récepteur. Thèse de doctorat en sciences de l'université René Descartes Paris V, école doctorale «Médicament, toxicologie, chimie et environnement», co-direction avec Dr. X. Iturrioz, mention très honorable avec les félicitations du jury.

\title{
Dynamique et physiopathologie des réseaux neuronaux (CIRB)
}

\author{
Responsable : Laurent VENANCE \\ Équipe : C. Bosch, Y. CuI, B. Degos, E. Fino, Y. GioAnNi, M.-L. Kemel, S. Pérez, \\ S. Valtcheva, M. Vandecasteele, H. Xu, L. Venance
}

\section{RECHERCHE}

Nous étudions les modalités d'encodage de la mémoire au niveau des ganglions de la base, un ensemble de structures cérébrales sous-corticales impliquées dans le contrôle adaptatif du comportement et la mémoire procédurale. Le striatum, le principal noyau d'entrée des ganglions de la base, reçoit des afférences de l'ensemble des aires du cortex cérébral. Les neurones de sortie striataux, silencieux au repos, doivent, pour décharger, recevoir des signaux corticaux de fortes amplitudes et synchrones. Ainsi, les neurones striataux agissent comme des détecteurs de coïncidence de l'activité corticale : ils extraient du bruit de fond les informations corticales jugées pertinentes et ce, en fonction du contexte (motivation, attention, etc.). L'intégration des informations corticales est fortement modulée par les afférences dopaminergiques de la substance noire pars compacta. Le rôle prépondérant de la dopamine est dramatiquement illustré par la maladie de Parkinson causée par la dégénérescence des neurones dopaminergiques. L'apprentissage et la mémorisation sont principalement sous-tendus par des phénomènes de plasticité synaptique à long-terme qui soit augmentent l'efficacité synaptique (potentialisation à long-terme, LTP), soit la diminuent (dépression à long-terme, LTD). La connaissance des modalités de la plasticité cortico-striatale est fondamentale pour comprendre les mécanismes qui sous-tendent le rôle des ganglions de la base dans l'apprentissage procédural et leurs altérations en conditions physiopathologiques.

\section{La « spike-timing dependent plasticity » cortico-striatale}

Nous avons utilisé des protocoles de «spike-timing dependent plasticity » (STDP), récemment décrits comme étant plus physiologiques et considérés actuellement comme la loi «élémentaire» de plasticité synaptique. La STDP repose sur un paradigme d'apprentissage Hebbien et donc sur la quasi-coïncidence entre des stimulations pré- et post-synaptique. Ainsi, il a été décrit dans différentes structures 